\title{
Fuzzy Spatial Relationships from Mathematical Morphology for Model-Based Pattern Recognition and Spatial Reasoning
}

\author{
Isabelle Bloch \\ Ecole Nationale Supérieure des Télécommunications \\ Dept. TSI - CNRS UMR 5141 \\ 46 rue Barrault, 75013 Paris, France \\ Isabelle.Bloch@enst.fr
}

\begin{abstract}
This paper discusses the interest of fuzzy set representations and of mathematical morphology for structural spatial knowledge representation and its use in model-based pattern recognition in images. It also briefly addresses the issues of digitization effects and computational aspects.
\end{abstract}

\section{Introduction}

In model-based pattern recognition in images, the model constitutes a description of the scene where objects have to be recognized. This description can be iconic, as for instance a digital map or a digital anatomical atlas, or symbolic, as a linguistic description of the main structures. The model can be attached to a specific scene, the typical example being a digital map used for recognizing structures in an aerial or satellite image of a specific region. It can also be more generic, as an anatomical atlas, which is a schematic representation that can be used for recognizing structures in a medical image of any person. In both types of descriptions (iconic and symbolic), objects are usually described through some characteristics like shape, size, appearance in the images, etc. But this is generally not enough to discriminate all objects in the scene, in particular if they are embedded in a complex environment. For instance in a magnetic resonance image (MRI) of the brain, several internal structures appear as smooth shapes with similar grey levels, making their individual recognition difficult. Similar examples can be found in other application domains. In such cases, spatial relationships play a crucial role, and it is important to include them in the model in order to guide the recognition. Such relationships can be of topological nature (inclusion, exclusion, adjacency...) or of metric nature (distances, orientations...). They often allow to identify structures that could not be distinguished based on their individual characteristics, by using their relationships to other structures.

This topic is the main component of spatial reasoning. Unlike temporal reasoning, which is well structured [1], spatial reasoning is more recent and less unified. It can be defined as the domain of spatial knowledge representation, in 
particular spatial relations between spatial entities, and of reasoning on these relations.

Usually vision and image processing make use of quantitative representations of spatial relationships. In artificial intelligence, mainly symbolic representations are developed (see [30] for a survey). The limitations of purely qualitative reasoning have already been stressed in [18, as well as the interest of adding semi-quantitative extension to qualitative value (as done in the fuzzy set theory for linguistic variables [3116]) for deriving useful and practical conclusions (as for recognition). On the other hand, purely quantitative representations are limited in the case of imprecise statements, and of knowledge expressed in linguistic terms. The use of fuzzy approaches for representing spatial relationships allows us to integrate both quantitative and qualitative knowledge, using the semi-quantitative interpretation of fuzzy sets. As already mentioned in [20], this allows to provide a computational representation and interpretation of imprecise spatial relations, expressed in a linguistic way, possibly including quantitative knowledge. These representations can then be used for semi-quantitative reasoning, intermediate between quantitative and qualitative spatial reasoning.

In this context, mathematical morphology is of particular interest. Although its basic transformations rely mainly on local information, based on the concept of structuring element, mathematical morphology also deals with more global and structural information since several spatial relationships can be expressed in terms of morphological operations (mainly dilations). Its algebraic framework leads to nice extensions to fuzzy sets with good properties, based on fuzzy mathematical morphology 9 .

In Section 2, we show how spatial relationships can be defined from mathematical morphology in the fuzzy set framework. This summarizes our previous work, and more details can be found in [10,64,7] for instance. In Section 3. we briefly explain how these relationships can be used in model-based pattern recognition. In Section 4, we mention some digital aspects and address computational issues.

\section{Fuzzy Spatial Relationships Based on Mathematical Morphology}

In this Section we address the problem of modeling spatial relationships in the fuzzy set framework. This framework is interesting here for several reasons:

- the objects of interest can be imprecisely defined, for instance due to the segmentation step;

- some relations are imprecise, such as to be left of, and find a more suitable definition in the fuzzy set framework;

- the type of knowledge available about the structures or the type of question we would like to answer can be imprecise too.

We consider here set relations, adjacency, distances, and directional relative position. Some of them have led to a rich literature in the fuzzy set community, 
like distances which have been defined using a lot of different approaches, while others have not raised so much attention. We summarize here our work based on fuzzy mathematical morphology [9], which allows us to represent in a unified way various spatial relationships [7].

Two types of questions are important for applications in structural pattern recognition:

1. given two object (possibly fuzzy) assess the degree to which a relation is satisfied;

2. given one reference object, define the area of the space in which a relation to this reference is satisfied (to some degree).

Our approach provides answers to these two types of questions. The second one will be illustrated only for distances and directional position here (see 7 for the other relations).

We consider the general case of a $3 \mathrm{D}$ space $\mathcal{S}$ (typically $\mathbb{R}^{3}$ or $\mathbb{Z}^{3}$ in the digital case), where objects can have any shape and any topology, and can be crisp or fuzzy.

Fuzzy Dilation and Erosion. Several definitions of fuzzy mathematical morphology have been proposed. Here we just give an example, chosen for its nice properties with respect to classical morphology, where dilation and erosion of a fuzzy set $\mu$ by a structuring element $\nu$ are respectively defined, for all $x \in \mathcal{S}$, by $[9$ :

$$
\begin{aligned}
& D_{\nu}(\mu)(x)=\sup \{t[\nu(y-x), \mu(y)], y \in \mathcal{S}\}, \\
& E_{\nu}(\mu)(x)=\inf \{T[c(\nu(y-x)), \mu(y)], y \in \mathcal{S}\} .
\end{aligned}
$$

where $t$ is a t-norm and $T$ the associated t-conorm with respect to the complementation $c$.

Set Relationships. If the objects are imprecise and represented as spatial fuzzy sets (i.e. fuzzy sets defined by membership functions from the spatial domain $\mathcal{S}$ into $[0,1])$, stating if they intersect or not, or if one is included in the other, becomes a matter of degree.

The degree of intersection between two fuzzy sets $\mu$ and $\nu$ can be defined using a supremum of a t-norm $t$ :

$$
\mu_{i n t}(\mu, \nu)=\sup _{x \in \mathcal{S}} t[\mu(x), \nu(x)]
$$

or using the fuzzy volume of the t-norm in order to take more spatial information into account:

$$
\mu_{i n t}(\mu, \nu)=\frac{V_{n}[t(\mu, \nu)]}{\min \left[V_{n}(\mu), V_{n}(\nu)\right]},
$$

where

$$
V_{n}(\mu)=\sum_{x \in \mathcal{S}} \mu(x)
$$


The degree of non-intersection is then simply defined by $\mu_{\neg \text { int }}=1-\mu_{\text {int }}$. It is interesting to note that the degree of intersection defined from a t-norm corresponds to the dilation of $\mu$ by $\nu$ at origin.

In a similar way, the degree of inclusion of $\nu$ in $\mu$ can be defined as:

$$
\inf _{x \in \mathcal{S}} T[c(\nu(x)), \mu(x)],
$$

and corresponds to the erosion of $\mu$ by $\nu$ at origin. These morphological interpretations allow us to include set relationships in the same framework as the other relations that will be detailed below.

Adjacency. Adjacency has a large interest in image processing and pattern recognition, since it denotes an important relationship between image objects or regions 29], widely used as a feature in model-based pattern recognition. In the crisp case, it is defined based on the digital connectivity $n_{c}(x, y)$ defined on the image: two subsets $X$ and $Y$ in $\mathcal{S}$ are adjacent according to the c-connectivity if: $X \cap Y=\emptyset$ and $\exists x \in X, \exists y \in Y: n_{c}(x, y)$. This definition can also be expressed equivalently in terms of morphological dilation, as: $X \cap Y=\emptyset$ and $D_{B}(X) \cap Y \neq \emptyset, \quad D_{B}(Y) \cap X \neq \emptyset$, where $B$ denotes the elementary structuring element associated to the c-connectivity.

This morphological expression can be extended to the fuzzy case, leading to the following degree of adjacency between two fuzzy sets [10]:

$$
\mu_{\text {adj }}(\mu, \nu)=t\left[\mu_{\neg i n t}(\mu, \nu), \mu_{i n t}\left[D_{B}(\mu), \nu\right], \mu_{i n t}\left[D_{B}(\nu), \mu\right]\right] .
$$

This definition represents a conjunctive combination (through a t-norm $t$ ) of a degree of non-intersection $\mu_{\neg i n t}$ between $\mu$ and $\nu$ and a degree of intersection $\mu_{\text {int }}$ between one fuzzy set and the dilation of the other. $B$ can be taken as the elementary structuring element related to the considered connectivity, or as a fuzzy structuring element, representing for instance spatial imprecision (i.e. the possibility distribution of the location of each point). We proved that this definition is symmetrical, consistent with the binary definition if $\mu, \nu$ and $B$ are binary, decreases if the distance between $\mu$ and $\nu$ increases, and is invariant with respect to geometrical transformations [10].

Distances. The importance of distances in image processing is well established. Their extensions to fuzzy sets can be useful in several parts of image processing under imprecision (classification and clustering, skeletonization, registration, structural pattern recognition, since distances constitute a major component of the spatial arrangement of objects).

Several definitions can be found in the literature for distances between fuzzy sets (which is the main addressed problem). They can be roughly divided in two classes: distances that take only membership functions into account and that compare them point-wise, and distances that additionally include spatial distances. The definitions which combine spatial distance and fuzzy membership comparison allow for a more general analysis of structures in images, for applications where topological and spatial arrangement of the structures of interest 
is important (segmentation, classification, scene interpretation). These distances combine membership values at different points in the space, and take into account their proximity or distance in $\mathcal{S}$. The price to pay is an increased complexity, generally quadratic in the cardinality of $\mathcal{S}$.

We proposed in [6] original approaches for defining fuzzy distances taking into account spatial information, which are based on fuzzy mathematical morphology. They are summarized below. The idea is that in the binary case, there exist strong links between mathematical morphology (in particular dilation) and distances (from a point to a set, and between two sets), and this can also be exploited in the fuzzy case. The advantage is that distances are expressed in set theoretical terms, and are therefore easier to translate to the fuzzy case with nice properties than usual analytical expressions. We just give the example of nearest point distance between two fuzzy sets. The case of Hausdorff distance can be treated in a similar way.

The minimum or nearest point distance between $X$ and $Y$ is defined (in the discrete finite case) as:

$$
d_{N}(X, Y)=\min _{(x, y) \in X \times Y} d_{E}(x, y)=\min _{x \in X} d_{E}(x, Y)=\min _{y \in Y} d_{E}(y, X),
$$

where $d_{E}$ denotes the Euclidean distance in $\mathcal{S}$. This has an equivalent morphological expression:

$$
d_{N}(X, Y)=\inf \left\{n \in \mathbb{N}, X \cap D^{n}(Y) \neq \emptyset\right\}=\inf \left\{n \in \mathbb{N}, Y \cap D^{n}(X) \neq \emptyset\right\} .
$$

By translating Equation 9, we define a distance distribution $\Delta_{N}\left(\mu, \mu^{\prime}\right)(n)$ that expresses the degree to which the distance between $\mu$ and $\mu^{\prime}$ is less than $n$ by:

$$
\Delta_{N}\left(\mu, \mu^{\prime}\right)(n)=f\left[\sup _{x \in \mathcal{S}} t\left[\mu(x), D_{\nu}^{n}\left(\mu^{\prime}\right)(x)\right], \sup _{x \in \mathcal{S}} t\left[\mu^{\prime}(x), D_{\nu}^{n}(\mu)(x)\right]\right],
$$

where $f$ is a symmetrical function.

A distance density, i.e. a fuzzy number $\delta_{N}\left(\mu, \mu^{\prime}\right)(n)$ representing the degree to which the distance between $\mu$ and $\mu^{\prime}$ is equal to $n$, can be obtained implicitly by $\Delta_{N}\left(\mu, \mu^{\prime}\right)(n)=\int_{0}^{n} \delta_{N}\left(\mu, \mu^{\prime}\right)\left(n^{\prime}\right) d n^{\prime}$. Clearly, this expression is not very tractable and does not lead to a simple explicit expression of $\delta_{N}\left(\mu, \mu^{\prime}\right)(n)$. Therefore, we suggest to use an explicit method, exploiting the fact that, for $n>0$, the nearest point distance can be expressed in morphological terms as:

$$
d_{N}(X, Y)=n \Leftrightarrow D^{n}(X) \cap Y \neq \emptyset \text { and } D^{n-1}(X) \cap Y=\emptyset
$$

or equivalently by the symmetrical expression. For $n=0$ we have $d_{N}(X, Y)=$ $0 \Leftrightarrow X \cap Y \neq \emptyset$. The translation of these equivalences provides, for $n>0$, the following distance density:

$$
\delta_{N}\left(\mu, \mu^{\prime}\right)(n)=t\left[\sup _{x \in \mathcal{S}} t\left[\mu^{\prime}(x), D_{\nu}^{n}(\mu)(x)\right], c\left[\sup _{x \in \mathcal{S}} t\left[\mu^{\prime}(x), D_{\nu}^{n-1}(\mu)(x)\right]\right]\right]
$$

or a symmetrical expression derived from this one, and $\delta_{N}\left(\mu, \mu^{\prime}\right)(0)=$ $\sup _{x \in \mathcal{S}} t\left[\mu(x), \mu^{\prime}(x)\right]$. 
This definition of fuzzy nearest point distances (defined as fuzzy numbers) between two fuzzy sets (and of Hausdorff distance too) does not necessarily share the same properties as their crisp equivalent, depending on the choice of the involved t-norms and t-conorms for some of them.

Let us now consider the second question, i.e. defining the area of the space that satisfies some distance property with respect to a reference object. We assume that a set $A$ is known as one already recognized object, or a known area of $\mathcal{S}$, and that we want to determine $B$, subject to satisfy some distance relationship with $A$. According to the algebraic expressions of distances, dilation of $A$ is an adequate tool for this. For instance if the knowledge expresses that $d(A, B) \geq n$, then $B$ should be looked for in $D^{n-1}(A)^{C}$. As another example, expressing that $B$ should lay between a distance $n_{1}$ and a distance $n_{2}$ of $A$ can be obtained by considering both minimum and maximum (Hausdorff) distances: the minimum distance should be greater than $n_{1}$ and the maximum distance should be less than $n_{2}$. In this case, the volume of interest for $B$ is reduced to $D^{n_{2}}(A) \backslash D^{n_{1}-1}(A)$.

In cases where imprecision has to be taken into account, fuzzy dilations are used, with the corresponding equivalences with fuzzy distances 96. The extension to approximate distances calls for fuzzy structuring elements. We define these structuring elements through their membership function $\nu$ on $\mathcal{S}$. Structuring elements with a spherical symmetry can typically be used, where the membership degree only depends on the distance to the center of the structuring element.

Let us consider the generalization to the fuzzy case of the last case (minimum distance of at least $n_{1}$ and maximum distance of at most $n_{2}$ to a fuzzy set $\mu$ ). Instead of defining an interval $\left[n_{1}, n_{2}\right]$, we consider a fuzzy interval, defined as a fuzzy set on $\mathbb{R}^{+}$having a core equal to the interval $\left[n_{1}, n_{2}\right]$. The membership function $\mu_{n}$ is increasing between 0 and $n_{1}$ and decreasing after $n_{2}$ (this is but one example). Then we define two structuring elements, as:

$$
\begin{gathered}
\nu_{1}(v)= \begin{cases}1-\mu_{n}\left(d_{E}(v, 0)\right) & \text { if } d_{E}(v, 0) \leq n_{1} \\
0 & \text { else }\end{cases} \\
\nu_{2}(v)= \begin{cases}1 & \text { if } d_{E}(v, 0) \leq n_{2} \\
\mu_{n}\left(d_{E}(v, 0)\right) & \text { else }\end{cases}
\end{gathered}
$$

where $d_{E}$ is the Euclidean distance in $\mathcal{S}$ and $O$ the origin. The spatial fuzzy set expressing the approximate relationship about distance to $\mu$ is then defined as:

$$
\mu_{\text {distance }}=t\left[D_{\nu_{2}}(\mu), 1-D_{\nu_{1}}(\mu)\right]
$$

if $n_{1} \neq 0$, and $\mu_{\text {distance }}=D_{\nu_{2}}(\mu)$ if $n_{1}=0$. The increasingness of fuzzy dilation with respect to both the set to be dilated and the structuring element 9 guarantees that these expressions do not lead to inconsistencies: we have $\nu_{1} \subset \nu_{2}$, $\nu_{1}(0)=\nu_{2}(0)=1$, and therefore $\mu \subset D_{\nu_{1}}(\mu) \subset D_{\nu_{2}}(\mu)$. In the case where $n_{1}=0$, we do not have $\nu_{1}(0)=1$ any longer, but in this case, only the dilation by $\nu_{2}$ is considered. This case corresponds actually to a distance to $\mu$ less than 
"about $n_{2}$ ". These properties are indeed expected for representations of distance knowledge.

Directional Relative Position. This type of relation is ambiguous and imprecise even of objects are crisp. Therefore, relative position concepts may find a better understanding in the framework of fuzzy sets, as fuzzy relationships, even for crisp objects. This framework makes it possible to propose flexible definitions which fit the intuition and may include subjective aspects, depending on the application and on the requirements of the user. The few existing fuzzy approaches in the literature mostly rely on angle histogram [2422] or extensions of it 23. Our approach is completely different since it works directly in the spatial domain.

Let us consider a reference object $R$ and an object $A$ for which the relative position with respect to $R$ has to be evaluated. In order to evaluate the degree to which $A$ is in some direction with respect to $R$, we propose the following approach 4, 5 :

1. We first define a fuzzy "landscape" around the reference object $R$ as a fuzzy set such that the membership value of each point corresponds to the degree of satisfaction of the spatial relation under examination.

2. We then compare the object $A$ to the fuzzy landscape attached to $R$, in order to evaluate how well the object matches with the areas having high membership values (i.e. areas that are in the desired direction). This is done using a fuzzy pattern matching approach, which provides an evaluation as an interval instead of one number only. This makes another difference with respect to all the previous approaches, and, to our opinion, it provides a richer information about the considered relationship.

The first step answers to the second type of question, while the second one answers to the first type.

Let us detail the first step. In the 3D Euclidean space $\mathcal{S}$, a direction is defined by two angles $\alpha_{1}$ and $\alpha_{2}$, where $\alpha_{1} \in[0,2 \pi]$ and $\alpha_{2} \in\left[-\frac{\pi}{2}, \frac{\pi}{2}\right]\left(\alpha_{2}=0\right.$ in the $2 \mathrm{D}$ case). The direction in which the relative position of an object with respect to another one is evaluated is denoted by: $\boldsymbol{u}_{\alpha_{1}, \alpha_{2}}=\left(\cos \alpha_{2} \cos \alpha_{1}, \cos \alpha_{2} \sin \alpha_{1}, \sin \alpha_{2}\right)^{t}$, and we note $\alpha=\left(\alpha_{1}, \alpha_{2}\right)$.

We denote by $\mu_{\alpha}(A)$ the fuzzy region representing the relation to be in the direction $\boldsymbol{u}_{\alpha_{1}, \alpha_{2}}$ with respect to reference object $A$. Points that satisfy this relation with high degrees should have high membership values. In other terms, the membership function $\mu_{\alpha}(A)$ has to be an increasing function of the degree of satisfaction of the relation.

Let us denote by $P$ any point in $\mathcal{S}$, and by $Q$ any point in $A$. Let $\beta(P, Q)$ be the angle between the vector $\boldsymbol{Q P}$ and the direction $\boldsymbol{u}_{\alpha_{1}, \alpha_{2}}$, computed in $[0, \pi]$ :

$$
\beta(P, Q)=\arccos \left[\frac{\boldsymbol{Q P} \cdot \boldsymbol{u}_{\alpha_{1}, \alpha_{2}}}{\|\boldsymbol{Q P}\|}\right] \text {, and } \beta(P, P)=0 .
$$

Setting $\beta(P, P)=0$ allows actually to deal with overlapping objects or with fuzzy objects with overlapping supports. 
We then determine for each point $P$ the point $Q$ of $A$ leading to the smallest angle $\beta$, denoted by $\beta_{\text {min. }}$. In the crisp case, this point $Q$ is the reference object point from which $P$ is visible in the direction the closest to $\boldsymbol{u}_{\alpha_{1}, \alpha_{2}}: \beta_{\min }(P)=$ $\min _{Q \in A} \beta(P, Q)$.

The spatial fuzzy set $\mu_{\alpha}(A)$ at point $P$ is then defined as $\mu_{\alpha}(A)(P)=$ $f\left(\beta_{\min }(P)\right)$, where $f$ is a decreasing function of $[0, \pi]$ into $[0,1]$. We choose a function that sets the values of $\mu_{\alpha}(A)(P)$ to 0 as soon as $\beta_{\text {min }}$ becomes greater than $\pi / 2$. This avoids to get positive membership values for points having coordinates completely outside of the coordinate range of $A$ in the desired direction.

In the fuzzy case, we propose a method which translates binary equations and propositions into fuzzy ones as:

$$
\mu_{\alpha}(A)(P)=\max _{Q \in \operatorname{Supp}(A)} t\left[\mu_{A}(Q), f(\beta(P, Q))\right]
$$

where $t$ is a t-norm.

An advantage of this approach is its easy interpretation in terms of morphological operations. It can indeed be shown [4] that $\mu_{\alpha}(A)$ is exactly the fuzzy dilation of $A$ by $\nu$, where $\nu$ is the fuzzy structuring element defined on $\mathcal{S}$ as:

$$
\forall P \in \mathcal{S}, \nu(P)=f[\beta(O, P)]
$$

with $O$ as the center of the structuring element.

Among the nice properties of this definition is invariance with respect to geometrical transformations (translation, rotation, scaling), which is a requirement in object recognition. It also has a behavior that fits well the intuition if the distance to the reference object increases, and in case of concavities. These properties are detailed in 4], and several examples are shown.

For the second step, let us denote by $\mu_{A}$ the membership function of the object $A$, which is a function of $\mathcal{S}$ into $[0,1]$. The evaluation of relative position of $A$ with respect to $R$ is given by a function of $\mu_{\alpha}(R)(x)$ and $\mu_{A}(x)$ for all $x$ in $\mathcal{S}$. An appropriate tool for defining this function is the fuzzy pattern matching approach [17]. Following this approach, the evaluation of the matching between two possibility distributions consists of two numbers, a necessity degree $N$ (a pessimistic evaluation) and a possibility degree $\Pi$ (an optimistic evaluation), as often used in the fuzzy set community. An average measure can also be useful from a practical point of view.

We proved [5] that the possibility has a symmetry property (i.e. the possibility for $A$ to be in some direction from $B$ is equal to the possibility of $B$ to be in the opposite direction with respect to $A$ ). Also, the proposed definition is invariant with respect to translation, rotation and scaling, for $2 \mathrm{D}$ and $3 \mathrm{D}$ objects (crisp and fuzzy). We also proved that when the distance between the objects increases, the objects are seen as points. The value of their relative position can be predicted only from the direction of interest and the direction in which one object goes far away from the reference object. Therefore the shape of the objects does no longer play any role in the assessment of their relative position. Finally, we looked at the behavior of the proposed definition in cases where the reference object 
has strong concavities, and show that the behavior corresponds to what can be intuitively expected.

\section{Use of Fuzzy Spatial Relationships in Model-Based Pattern Recognition}

Let us now briefly illustrate how these fuzzy spatial relations can be used for recognizing structures in a scene based on a model of this scene. Two types of approaches can be developed, corresponding to the two types of questions mentioned in Section 2

Graph-Based Approach. In the first approach, spatial relations evaluated between spatial entities (typically objects or regions) are considered as attributes in a graph.

Graph representations are widely used for dealing with structural information, in different domains including image interpretation and model-based pattern recognition. Here, we assume that the model is represented as a graph where nodes are objects and edges represent links between these objects. Both nodes and edges are attributed. Node attributes are characteristics of the objects, while edge attributes quantify spatial relationships between the objects. A data graph is then constructed from each image where the recognition has to be performed. Since it is usually difficult to segment directly the objects, usually the graph is based on an over-segmentation of the image, for instance based on watersheds. Each region constitutes a node of this data graph, and edges represent links between regions. Attributes are computed as for the model. The use of fuzzy relations is particularly useful in order to be less sensitive to the segmentation.

One important problem to be solved then is graph matching. In order to achieve a good correspondence between both graphs, the most used concept is the one of graph isomorphism and a lot of work is dedicated to the search for the best isomorphism between two graphs or subgraphs. However, in a number of cases, the bijective condition is too strong: because of the schematic aspect of the model and of the difficulty to segment the image into meaningful entities, no isomorphism can be expected between both graphs. In particular, several regions of the image can be assigned to the same node of the model graph. Such problems call for inexact graph matching. It constitutes generally in finding a morphism, which furthermore optimizes an objective function based on similarities between attributes. The morphism aims an preserving the structure of the graphs, while the objective function privileges the association between nodes, respectively between edges, with similar attribute values. This approach can benefit from the huge literature on fuzzy comparison tools (see e.g. [13]) and from recent developments on fuzzy morphisms 25]. The optimization is not an easy task since the problem is NP-hard. Genetic algorithms, estimation of distribution algorithms (EDA) and tree search methods have been developed towards this aim [26|2 14].

This approach has been applied in brain imaging, in order to recognize brain structures in a 3D magnetic resonance image (MRI) based on an anatomical at- 


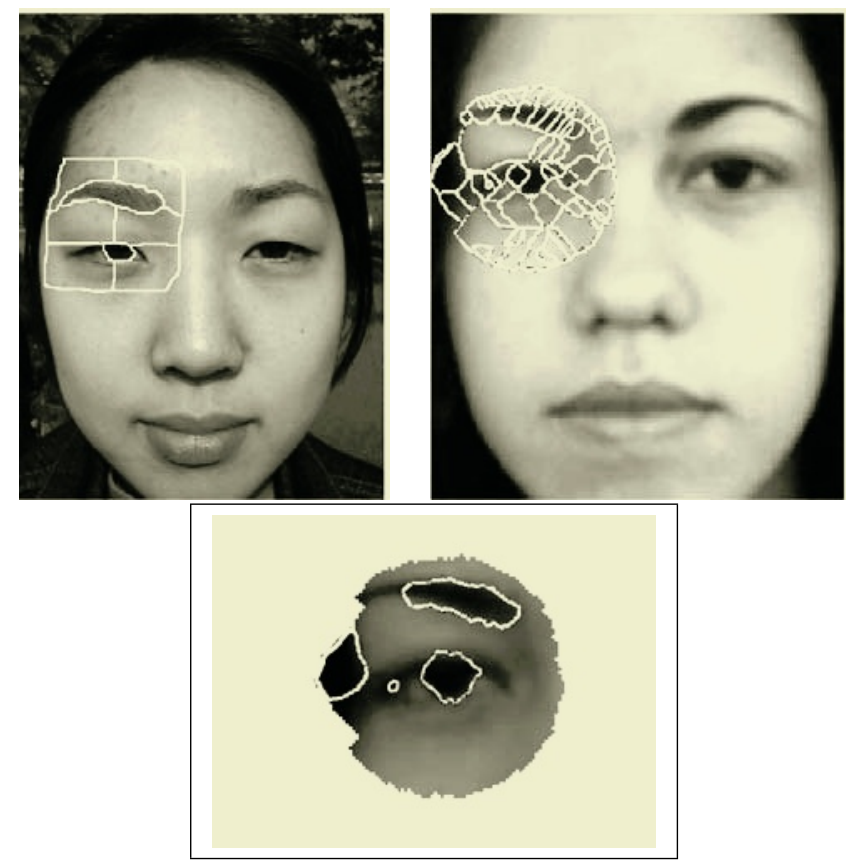

Fig. 1. Left: model; middle: over-segmented image (subset); bottom: results on a few face features obtained with EDA (from [14]).

las, and in face feature recognition, based on a rough model of a face constructed from a different person image (an example is shown in Figure 1).

Focusing Attention Based on Spatial Representation of Spatial Knowledge. In the second type of approach, we use the spatial representation of spatial knowledge. Each relation is then represented as a spatial fuzzy set, constraining the search for the object that should satisfy this relation. This region of interest allows to focus attention towards the only region satisfying the relation (to some degree). Since usually several relations are represented in the model for describing one structure, fusion of these representations should be performed. The fuzzy set frameworks offers a large set of fusion operators, varying from conjunctive to disjunctive ones, including adaptive operators [3]. The fusion of all regions of interest leads to a fuzzy region representing the combination of all relationships concerning one structure. Then segmentation of the structure can be based on the image information (typically grey levels) focused in the obtained fuzzy region.

A recognition procedure based on this type of representation has been developed for the recognition of internal brain structures in MRI 218]. The model has an iconic part (digital atlas) and a symbolic part (linguistic descriptions of relationships between anatomical structures). The procedure consists in recognizing first simple structures (typically brain and lateral ventricles), and then progressively more and more difficult structures, based on relationships between these 

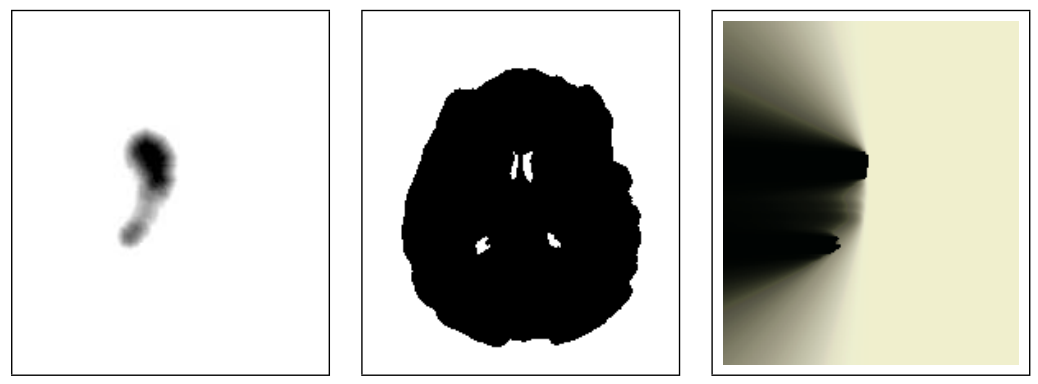

Fig. 2. Information representation in the image space (only one slice of the $3 \mathrm{D}$ volume is shown), illustrating knowledge about one caudate nucleus: shape information (left), set relationships (middle), and relative directional relationship (right). Membership values vary from 0 (white) to 1 (black).

structures and previously recognized structures. Each relationship describing the structure to be recognized is translated into a spatial fuzzy set representing the area satisfying this relation, to some degrees. The fuzzy sets representing all relationships involved in the recognition processed are fused using a numerical fusion operator. In the obtained fuzzy region of interest, a segmentation procedure is performed, and the quality of the results is guaranteed by the very restricted (focused) area in which the structure of interest is searched.

For instance, the recognition of a caudate nucleus in a 3D MRI image uses the previous recognition of brain and lateral ventricles and the following pieces of knowledge, illustrated in Figure 2

- rough shape and localization are provided by the representation of the caudate nucleus in the atlas, and its fuzzy dilation to account for variability and for inexact matching between the model and the image,

- the caudate nucleus belongs to the brain (black) but is outside from both lateral ventricles (white components inside the brain),

- the caudate nucleus is lateral to the lateral ventricle.

These pieces of knowledge can be combined (also with information extracted from the image itself), which leads to a successful recognition of the caudate nucleus.

Figure 3 illustrates the spatial representation of some knowledge about distances, used for other structures.

Figure 4 shows 3D views of some cerebral objects as defined in the atlas and as recognized in an MR image with our method. They are correctly recognized although the size, the location and the morphology of these objects in the image significantly differ from their definitions in the atlas. Note in particular the good recognition of third and fourth ventricles, that are very difficult to segment directly from the image. Here the help of relationships to other structures is very important. 


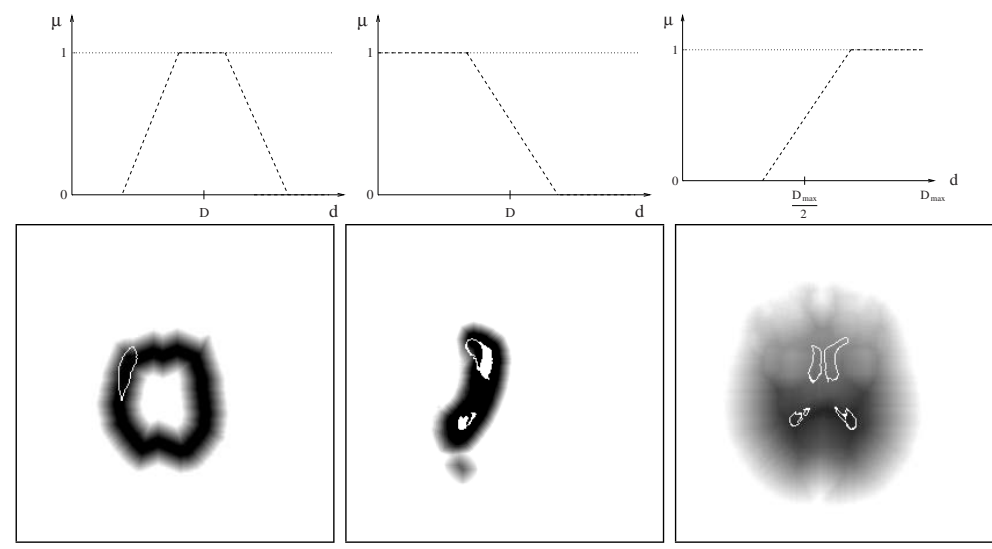

Fig. 3. Examples of representation of knowledge about distances. Top: membership functions $\mu_{n}$. Bottom: spatial fuzzy sets. The following types of knowledge are illustrated: the putamen has an approximately constant distance to the brain surface (left), the caudate nucleus is at a distance about less than $D$ from the lateral ventricles (in white) (middle), lateral ventricles are inside the brain and at a distance larger than about $D$ from the brain surface (right). The contours of the o bjects we are looking at are shown in white.
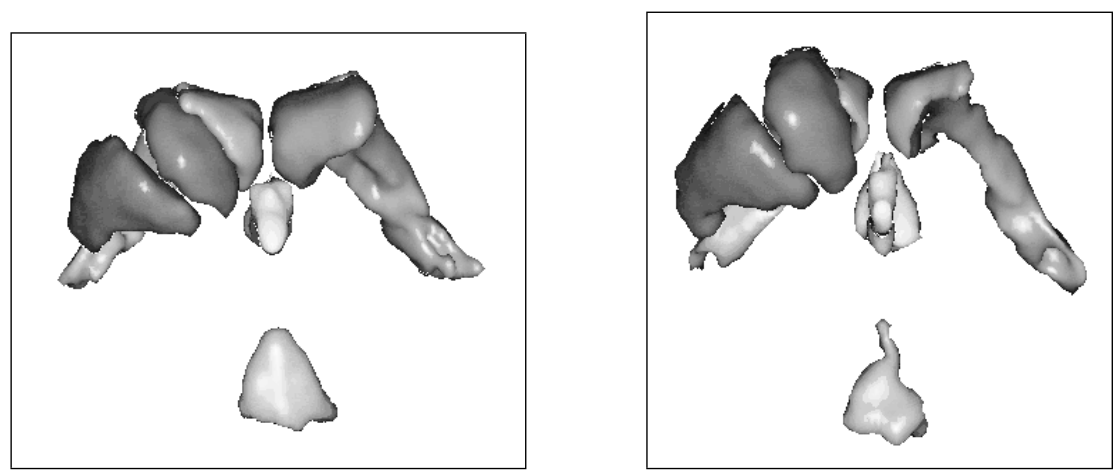

Fig. 4. Recognition results. The left view represents six objects from the model atlas: lateral ventricles (medium grey), third and fourth ventricles (light grey), caudate nucleus and putamen (dark grey). The right view represents the equivalent objects recognized from a MRI acquisition. (From [21].)

The segmentation can be further improved once recognition is achieved by integrating the fuzzy regions representing the spatial relations as new energy terms in deformable models [15.

This approach has been used in other domains, for instance in mobile robotics to reason about the spatial position of the robot and the structure of its environment 11. 

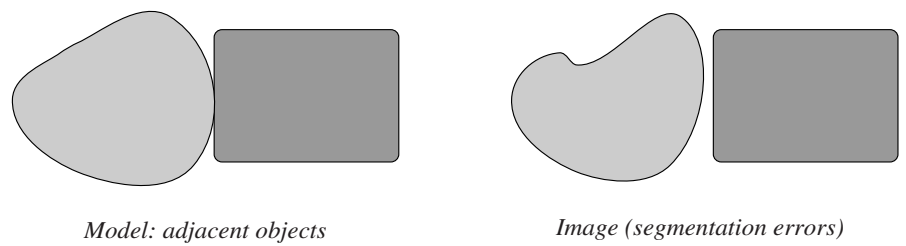

Fig. 5. Sensitivity of crisp adjacency: small modifications in the shapes may completely change the adjacency relation, and thus prevent a correct recognition based on this relationship.

\section{Digital Aspects and Computational Issues}

In this Section we address a few issues related to digital and computational aspects. In particular we show that introducing fuzziness overcomes some problems occurring when working in digital spaces. Although this may induce additional computation cost, some fast algorithms can be designed to get good approximations in reasonable time.

Topological Relations. We first discuss set relationships and adjacency. In digital spaces, these relations are highly sensitive, since in the binary case, the result can depend on one point only. Also the segmentation can induce errors that can completely change the relations. For instance two objects that are expected to be adjacent (for instance if they are described as such in the model) can appear as not adjacent depending on the digitization or if some even tiny errors occur during the segmentation. An example illustrates this sensitivity in Figure 5.

This is clearly a limitation of binary (all or nothing) definitions.

In the fuzzy case, the problem is much less crucial. Indeed, there is no more strict membership, the fuzziness allows us to deal with some gradual transition between objects or between object and background, and relations become then a matter of degree. Therefore, through the notions of fuzzy neighborhood, fuzzy inclusion and fuzzy adjacency, we can expect to gain in robustness when assessing the relationships between two objects.

In this respect, the fuzziness, even on digital images, could be interpreted as a partial recovering of the continuity lost during the digitization process.

Two ways can be followed to achieve this aim, for instance for adjacency [10]. In the first one, the satisfaction of the adjacency property between two objects is considered to be a matter of degree even if the objects are crisp; this can be more appropriate than a binary index [27 28]. This degree can be a decreasing function of the minimum distance between the object for instance (a zero distance meaning exact adjacency). The second one consists in introducing imprecision in the objects themselves, and to deal with fuzzy spatial objects. For instance, spatial imprecision due to the limited quality of image information can be represented in an adequate way by considering fuzzy objects. Then obviously adjacency is also a matter of degree. This is the approach described in Section 2 


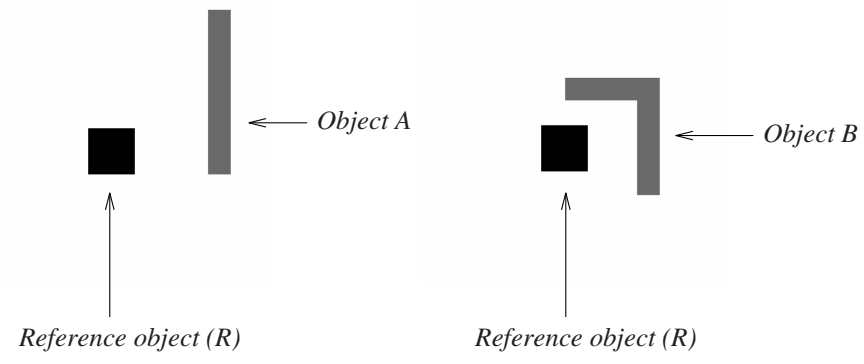

Fig. 6. Two examples where the relative position of objects with respect to the reference object is difficult to define in a "all-or-nothing" manner: the object $A$ is to the right of $R$ but it can also be considered to be to some extent above it; object $B$ is strongly to the right of $R$ and above it.

Relative Directional Position. The above discussion on robustness also holds for relative directional position.

An additional aspect concerns the digitization of directions of space. The approach described in Section 2 offers several advantages from this point of view. In particular it avoids to describe the relative position by only one dominant direction, which is not satisfactory in several situations, even of moderate complexity (see examples of Figure 6). It is also more flexible than just cardinal directions, which is an advantage of the semi-quantitative aspect.

But one limitation of this approach is that its computation might be a problem in large 3D spaces. Here we can take benefit of the digital nature of the space to propose two solutions to reduce the computational cost.

The computation can be made faster by storing the list of points in $R$ (which are often much less numerous than all image points), and by tabulating angles (since $\boldsymbol{Q P}$ takes a finite number of integer values in discrete images). The interpretation of the proposed definition as a fuzzy dilation may suggest a further way to reduce the computation time by reducing the precision of $\mu_{\alpha}(R)$ : it consists in performing the fuzzy dilation with a limited support for the structuring element. This amounts to have a rough quantification of angles, and therefore an approximate result is obtained.

A second solution is to approximate the result using a propagation algorithm, similar as the ones used for computing chamfer distances. We have proposed in [4] a fast algorithm for computing $\mu_{\alpha}(R)$, that still provides an approximation of $\mu_{\alpha}(R)$ but with increased precision with respect to the algorithm based on dilation. This algorithm is based on a propagation technique inspired by chamfer methods used for instance for discrete distance computation 12 . This idea comes from the observation of thew results where it appears that membership values in the fuzzy set $\mu_{\alpha}$ are constant along lines issued from contour points of the reference object.

The algorithm consists in performing two passes on the image, one in the conventional sense, and one in the opposite sense. For each point $P$, we store the point $Q=O(P)$ from which the minimum visibility angle is obtained. For 
a point $P$, we do not consider all points in $R$ as for the exhaustive method, but only those of a neighborhood of $P$. The algorithm consists of the following steps:

1. Initialization: we set $O(P)=P$ if $P \in R$ and $O(P)=N$ ull otherwise.

2. First pass: we compute the fuzzy landscape from visibility angle at $P$ as: $\mu_{\alpha}(R)(P)=\max _{Q \in V(P)} t\left[\mu_{R}(O(Q)), f(\beta(P, O(Q)))\right]$, where $V(P)$ denotes the neighborhood of $P$. Let $Q_{P}$ be the point $Q$ for which the maximum value is obtained is obtained:

$Q_{P}=\arg \max _{Q \in V(P)} t\left[\mu_{R}(O(Q)), f(\beta(P, O(Q)))\right]$. Then we set: $O(P)=$ $O\left(Q_{P}\right)$.

3. Second pass: it is performed as the first one, except that the points are examined in the reverse order. Note that during these two passes, the points of $R$ can also be modified.

This algorithm is applicable in $2 \mathrm{D}$ as well as in $3 \mathrm{D}$, and for crisp objects as well as for fuzzy ones. We used 8-connectivity in 2D, and 26-connectivity in 3D for defining $V(P)$. More precise results could be obtained with larger neighborhoods or with more passes on the image using other propagation directions, but at the price of extended computation time. The errors are mainly due to the fact that when there are several candidates for $Q_{P}$ (i.e. leading to the same minimal value for $\left.\beta_{\min }\right)$, there is no clear strategy of choice of one particular point among the candidates.

Although the result obtained for $\mu_{\alpha}(R)$ using the propagation algorithm is not exact, it can be considered as a good approximation. Figure 7 illustrates the results obtained with the propagation algorithm and the difference with the exact method for several reference objects. They show the quality of the approximation. The results may show no error at all depending on the angle with respect to the propagation directions, and depending on the object (this is the case for instance for the square of Figure 6). In the fuzzy case too, only few differences can be observed. Moreover, when using these results instead of the exact ones, we observed only few differences in the pattern matching results (the maximum error is at most a few percentage points, and generally less than $5 \%$ ). These differences cannot be considered as of much significance for pattern recognition purposes.

Distances. As for distances, the possibility to represent distance knowledge expressed in an imprecise way offers more flexibility and more robustness against digitization effects. For instance stating that two objects are at a distance exactly 10 can easily fail due to the digitization. On the contrary, modeling a distance of "about 10" as illustrated in Figure 3 is much more robust.

Let us now address the computational aspects for distances. Here again we can take advantage of the digitization.

If the object is binary, we can compute a distance map to the object using a chamfer algorithm, as usual. Then the values of the fuzzy relation are obtained by using simply the curves of Figure 3 as a look-up-table. This is the approach used for instance in 21 . 

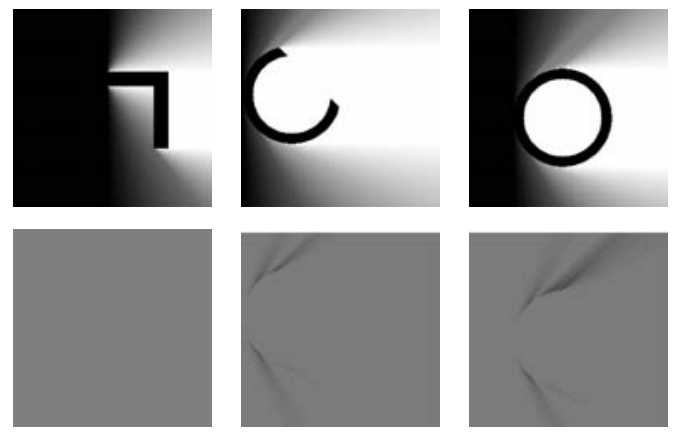

Fig. 7. A few examples of $\mu_{\alpha}(R)$ for $\alpha_{1}=\alpha_{2}=0$ for different types of reference objects (reference objects are black) using the propagation method. The second line shows the difference with the exact method (a grey level of 128 corresponds to no error, and the differences have been enhanced for the visualization). For the corner (left example) we obtain no error for all directions. (From [4].)

In the fuzzy case, an approximation of the distance map can be obtained by dilating the reference object by a fuzzy structuring element of conic shape, the membership values at each point being a linear function of the distance to the origin. In the binary case, this approach is exact, while it is only an approximation in the fuzzy case. Computation can be reduced by limiting the size of the support of the structuring element to some maximal interesting distance. This type of fuzzy structuring element has been used for instance in [19] to represent the concept of large open space in a robot's environment.

Finally, if we have a fuzzy object and a fuzzy distance knowledge representation, it could be interesting to develop algorithms generalizing chamfer algorithms to address the second type of question raised in Section 2 . We leave this for our future work.

\section{Conclusion}

The spatial arrangement of objects in images provides important information for recognition and interpretation tasks, in particular when the objects are embedded in a complex environment like in medical or remote sensing images. Such information can be expressed in different ways varying from purely quantitative and precise ones to purely qualitative and symbolic ones. The fuzzy set framework provides an interesting semi-quantitative alternative. We have shown that mathematical morphology provides an appropriate framework to express different types of spatial relationships in a unified formalism and to answer different questions about them, with good properties. Due to the strong algebraic structure of this framework, it applies to objects represented as sets, as fuzzy sets, and as logical formulas as well, which offers different points of view compared to the one adopted in this paper [7].

The different types of representation of spatial relations lead to model-based pattern recognition approaches such as graph-based or focalization methods. 
Applications of this work concern model-based pattern recognition in complex images, spatial knowledge representation issues, and spatial reasoning.

Finally digital and computational issues can also benefit from the fuzzy set framework, in particular to gain in robustness. These aspects are worth to be further developed in future work.

\section{References}

1. J. Allen. Maintaining Knowledge about Temporal Intervals. Comunications of the ACM, 26(11):832-843, 1983.

2. E. Bengoetxea, P. Larranaga, I. Bloch, A. Perchant, and C. Boeres. Inexact Graph Matching by Means of Estimation of Distribution Algorithms. Pattern Recognition, $35: 2867-2880,2002$.

3. I. Bloch. Information Combination Operators for Data Fusion: A Comparative Review with Classification. IEEE Transactions on Systems, Man, and Cybernetics, 26(1):52-67, 1996.

4. I. Bloch. Fuzzy Relative Position between Objects in Image Processing: a Morphological Approach. IEEE Transactions on Pattern Analysis and Machine Intelligence, 21(7):657-664, 1999.

5. I. Bloch. Fuzzy Relative Position between Objects in Image Processing: New Definition and Properties based on a Morphological Approach. International Journal of Uncertainty, Fuzziness and Knowledge-Based Systems, 7(2):99-133, 1999.

6. I. Bloch. On Fuzzy Distances and their Use in Image Processing under Imprecision. Pattern Recognition, 32(11):1873-1895, 1999.

7. I. Bloch. Mathematical Morphology and Spatial Relationships: Quantitative, SemiQuantitative and Symbolic Settings. In L. Sztandera and P. Matsakis, editors, Applying Soft Computing in Defining Spatial Relationships, pages 63-98. Physica Verlag, Springer, 2002.

8. I. Bloch, T. Géraud, and H. Maître. Representation and Fusion of Heterogeneous Fuzzy Information in the 3D Space for Model-Based Structural Recognition - Application to 3D Brain Imaging. Artificial Intelligence Journal, 2003.

9. I. Bloch and H. Maître. Fuzzy Mathematical Morphologies: A Comparative Study. Pattern Recognition, 28(9):1341-1387, 1995.

10. I. Bloch, H. Maître, and M. Anvari. Fuzzy Adjacency between Image Objects. International Journal of Uncertainty, Fuzziness and Knowledge-Based Systems, 5(6):615-653, 1997.

11. I. Bloch and A. Saffiotti. On the Representation of Fuzzy Spatial Relations in Robot Maps. In IPMU 2002, volume III, pages 1587-1594, Annecy, France, 2002.

12. G. Borgefors. Distance Transforms in the Square Grid. In H. Maître, editor, Progress in Picture Processing, Les Houches, Session LVIII, 1992, chapter 1.4, pages 46-80. North-Holland, Amsterdam, 1996.

13. B. Bouchon-Meunier, M. Rifqi, and S. Bothorel. Towards General Measures of Comparison of Objects. Fuzzy Sets and Systems, 84(2):143-153, September 1996.

14. R. Cesar, E. Bengoetxea, and I. Bloch. Inexact Graph Matching using Stochastic Optimization Techniques for Facial Feature Recognition. In International Conference on Pattern Recognition ICPR 2002, Québec, aug 2002.

15. O. Colliot. Représentation, évaluation et utilisation de relations spatiales pour l'interprétation d'images, application à la reconnaissance de structures anatomiques en imagerie médicale. PhD thesis, Ecole Nationale Supérieure des Télécommunications, 2003. 
16. D. Dubois and H. Prade. Fuzzy Sets and Systems: Theory and Applications. Academic Press, New-York, 1980.

17. D. Dubois, H. Prade, and C. Testemale. Weighted Fuzzy Pattern Matching. Fuzzy Sets and Systems, 28:313-331, 1988.

18. S. Dutta. Approximate Spatial Reasoning: Integrating Qualitative and Quantitative Constraints. International Journal of Approximate Reasoning, 5:307-331, 1991.

19. E. Fabrizi and A. Saffiotti. Extracting Topology-Based Maps from Gridmaps. In IEEE International Conference on Robotics and Automation (ICRA-2000), San Francisco, CA, 2000.

20. J. Freeman. The Modelling of Spatial Relations. Computer Graphics and Image Processing, 4(2):156-171, 1975.

21. T. Géraud, I. Bloch, and H. Maître. Atlas-guided Recognition of Cerebral Structures in MRI using Fusion of Fuzzy Structural Information. In CIMAF'99 Symposium on Artificial Intelligence, pages 99-106, La Havana, Cuba, 1999.

22. J. M. Keller and X. Wang. Comparison of Spatial Relation Definitions in Computer Vision. In ISUMA-NAFIPS'95, pages 679-684, College Park, MD, September 1995.

23. P. Matsakis and L. Wendling. A New Way to Represent the Relative Position between Areal Objects. IEEE Trans. on Pattern Analysis and Machine Intelligence, 21(7):634-642, 1999.

24. K. Miyajima and A. Ralescu. Spatial Organization in 2D Segmented Images: Representation and Recognition of Primitive Spatial Relations. Fuzzy Sets and Systems, 65:225-236, 1994.

25. A. Perchant and I. Bloch. Fuzzy Morphisms between Graphs. Fuzzy Sets and Systems, 128(2):149-168, 2002.

26. A. Perchant, C. Boeres, I. Bloch, M. Roux, and C. Ribeiro. Model-based Scene Recognition Using Graph Fuzzy Homomorphism Solved by Genetic Algorithm. In GbR'99 2nd International Workshop on Graph-Based Representations in Pattern Recognition, pages 61-70, Castle of Haindorf, Austria, 1999.

27. A. Rosenfeld. Fuzzy Digital Topology. Information and Control, 40:76-87, 1979.

28. A. Rosenfeld. The Fuzzy Geometry of Image Subsets. Pattern Recognition Letters, 2:311-317, 1984.

29. A. Rosenfeld and A. C. Kak. Digital Picture Processing. Academic Press, NewYork, 1976.

30. L. Vieu. Spatial Representation and Reasoning in Artificial Intelligence. In O. Stock, editor, Spatial and Temporal Reasoning, pages 5-41. Kluwer, 1997.

31. L. A. Zadeh. The Concept of a Linguistic Variable and its Application to Approximate Reasoning. Information Sciences, 8:199-249, 1975. 